\title{
Implementation of a First-Order ABC in Mixed Finite-Element Time-Domain Formulations Using Equivalent Currents
}

\author{
Ali Akbarzadeh-Sharbaf, Student Member, IEEE, and Dennis D. Giannacopoulos, Member, IEEE
}

\begin{abstract}
In this letter, we describe an easy approach to implement the first-order Bayliss-Turkel-like absorbing boundary condition (ABC) in two mixed finite-element time-domain (FETD) formulations, namely the Crank-Nicolson FETD (CN-FETD) and the leap-frog FETD (LF-FETD). The idea is to introduce a current source distribution on the outer boundary of the domain such that it cancels outgoing waves. The current distribution is obtained based on the $\mathrm{ABC}$ relation. In addition, we show that the CN-FETD and the LF-FETD are equivalent to the FETD based on the vector wave equation discretized by the Newmark- $\beta$ method in time with $\beta=1 / 4$ and 0 , respectively. Having utilized these equivalences, we demonstrate that our approach to implement the $\mathrm{ABC}$ in the mixed formulations lead to the same result as the vector wave FETD truncated with the same ABC. A numerical example is provided to validate our formulations.
\end{abstract}

Index Terms-Absorbing boundary conditions (ABCs), finite-element time-domain (FETD) method.

\section{INTRODUCTION}

T HE finite-element method (FEM) has been successfully applied to radiation and scattering problems [1], [2]. However, the computational domain has to be truncated in solving open-region problems. To truncate the domain, there are three main approaches, namely integral-equation methods, the perfectly-matched layer (PML) and absorbing boundary conditions $(\mathrm{ABCs})$. In the first approach, the exterior field is expressed in terms of a boundary integral known as FE-BI methods [3]. It is exact and can tightly enclose the structure under analysis. Although it has been recently extended to the time domain [4], [5], calculation of BI matrices is computationally expensive and the FE-BI matrices are no longer sparse. The PML was originally developed to truncate finite-difference time-domain (FDTD) grids. In contrast to the FDTD, PML formulation in FETD is much more complicated and numerical experiments have revealed late-time instabilities in the unconditionally stable (US) vector wave equation [6], [7]. Although PML applied to the conditionally stable (CS) LF-FETD do not suffer from instability issues [8], we expect the same problem in the PML for the CN-FETD due to the

Manuscript received January 29, 2013; revised March 25, 2013; accepted April 02, 2013. Date of publication April 26, 2013; date of current version June 03, 2013. This work was supported by the Natural Sciences and Engineering Research Council (NSERC) of Canada.

The authors are with the Department of Electrical and Computer Engineering, McGill University, Montreal, QC H3A 0E9, Canada (e-mail: ali.akbarzadehsharbaf@mail.mcgill.ca; dennis.giannacopoulos@mcgill.ca).

Color versions of one or more of the figures in this paper are available online at http://ieeexplore.ieee.org.

Digital Object Identifier 10.1109/LMWC.2013.2258462 underlying equivalence between this and the US vector wave equation shown in Section III. Alternatively, ABCs are easy to implement and do not destroy sparsity of the FEM matrices nor produce instabilities. The well-known and well-established Bayliss-Turkel ABC [9], [10] has been extended to arbitrary order of approximation for electromagnetic problems [11]. The first-order $\mathrm{ABC}$ is widely-used and the second-order has been recently implemented into the vector FEM effectively [12].

There are two main formulations for the FETD [13]. The first one discretizes the second-order vector wave equation; we call it VW-FETD, and the other is based on the two first-order Maxwell equations, known as a mixed formulation. The firstorder Bayliss-Turkel ABC can be easy applied to the former: invoking the vector Green's theorem produces a surface integral which can be utilized to implement the $\mathrm{ABC}$ in a straightforward manner. However, to the best knowledge of the authors, implementation of the $\mathrm{ABC}$ in a mixed formulation is not discussed in the literature. This may be because, in contrast to the VW-FETD, no appropriate surface integral appears in the deriving functional to facilitate implementation.

In this letter, a simple but effective approach is developed to implement the first-order ABC in the mixed FETD methods. It is based on applying an appropriate current distribution on the boundary as a source to reduce reflections. In addition, some useful equivalences between two kinds of formulations are shown.

\section{FORMULATION}

Consider the two first-order Maxwell equations on a domain $\Omega$ enclosed by an artificial boundary $\partial \Omega$, where $\hat{n}$ represents the outward unit normal vector and $\mathbf{J}_{i m p}$ denotes an arbitrary distribution of the electric current density inside $\Omega$ (excitation).

Expanding the electric field and the magnetic field variables in terms of edge (Whitney 1-form) and face elements (Whitney 2-form), respectively, yields [14]

$$
\begin{aligned}
{[\mathcal{M}] \frac{\partial\{e\}}{\partial t} } & =[\mathcal{C}]^{T}\left[\mathcal{M}_{f}\right]\{b\}-\left\{J_{i m p}\right\}-\left\{J_{e q u}\right\} \\
\frac{\partial\{b\}}{\partial t} & =-[\mathcal{C}]\{e\}
\end{aligned}
$$

where $\{e\}=\left[e_{1}, e_{2}, \ldots, e_{N_{e d}}\right]^{T}$ and $\{b\}=\left[b_{1}, b_{2}, \ldots, b_{N_{f}}\right]^{T}$ represent the electric field intensity and the magnetic flux density, respectively. The incident matrix $[\mathcal{C}]$ is a sparse and rectangular matrix consisting of $\{-1,0,+1\}$ entries and

$$
\begin{gathered}
{[\mathcal{M}]_{i j}=\int_{\Omega} \epsilon \boldsymbol{W}_{i}^{(1)} \cdot \boldsymbol{W}_{j}^{(1)} d \Omega} \\
{\left[\mathcal{M}_{f}\right]_{i j}=\int_{\Omega} \mu^{-1} \boldsymbol{W}_{i}^{(2)} \cdot \boldsymbol{W}_{j}^{(2)} d \Omega .}
\end{gathered}
$$


For the sake of clarity, we have assigned a different variable, $\boldsymbol{J}_{\text {equ }}$, to represent the equivalent current distribution on the external boundary utilized to implement the $\mathrm{ABC}$. We have

$$
\begin{aligned}
& \left\{J_{i m p}\right\}=\int_{\Omega} \boldsymbol{W}_{i}^{(1)} \cdot \boldsymbol{J}_{i m p} d \Omega \\
& \left\{J_{\text {equ }}\right\}=\int_{\partial \Omega} \boldsymbol{W}_{i}^{(1)} \cdot \boldsymbol{J}_{\text {equ }} d \Omega .
\end{aligned}
$$

Utilizing the leap-frog method to discretize (1) in time, and average value of $\boldsymbol{J}_{\text {equ }}$, one can reach

$$
\begin{aligned}
{[\mathcal{M}]\{e\}^{n+1}=} & {[\mathcal{M}]\{e\}^{n}+\Delta t[\mathcal{C}]^{T}\left[\mathcal{M}_{f}\right]\{b\}^{n+\frac{1}{2}} } \\
& -\frac{\Delta t}{2}\left(\{J\}_{\text {equ }}^{n+1}+\{J\}_{\text {equ }}^{n}\right) \\
\{b\}^{n+\frac{1}{2}}= & \{b\}^{n-\frac{1}{2}}-\Delta t[\mathcal{C}]\{e\}^{n}
\end{aligned}
$$

where $\left\{J_{i m p}\right\}$ is omitted for the sake of brevity. We call this formulation the LF-FETD.

On the other hand, we know that the first-order Bayliss-Turkel-like ABC for the electric field in the free-space is

$$
\hat{n} \times\left(\frac{1}{\mu_{0}} \nabla \times \boldsymbol{E}\right)+Y_{0} \hat{n} \times\left(\hat{n} \times \frac{\partial}{\partial t} \boldsymbol{E}\right) \approx 0
$$

where $Y_{0}=\sqrt{\epsilon_{0} / \mu_{0}}$. Invoking Faraday's law, one can obtain

$$
\frac{\partial \boldsymbol{J}_{e q u}}{\partial t} \approx Y_{0} \frac{\partial(\hat{n} \times \hat{n} \times \boldsymbol{E})}{\partial t}
$$

where $\boldsymbol{J}_{e q u}=\hat{n} \times \boldsymbol{H}(\boldsymbol{H}(\boldsymbol{r}), \boldsymbol{r} \in \partial \Omega)$ is the equivalent current on the boundary. Discretizing (8) at $t=(n+1 / 4) \Delta t$ and $t=$ $(n+3 / 4) \Delta t$ using the central-difference, which yields

$$
\begin{gathered}
\boldsymbol{J}_{e q u}^{n+1}-\boldsymbol{J}_{e q u}^{n+\frac{1}{2}}=Y_{0} \hat{n} \times \hat{n} \times\left(\boldsymbol{E}^{n+1}-\boldsymbol{E}^{n+\frac{1}{2}}\right) \\
\boldsymbol{J}_{e q u}^{n+\frac{1}{2}}-\boldsymbol{J}_{e q u}^{n}=Y_{0} \hat{n} \times \hat{n} \times\left(\boldsymbol{E}^{n+\frac{1}{2}}-\boldsymbol{E}^{n}\right)
\end{gathered}
$$

one can reach the following equation by simply subtracting them:

$$
\boldsymbol{J}_{e q u}^{n+1}+\boldsymbol{J}_{e q u}^{n}=Y_{0} \hat{n} \times \hat{n} \times\left(\boldsymbol{E}^{n+1}+\boldsymbol{E}^{n}\right) .
$$

Since the left hand side of (10) is applied as a current source, the negative of it should be substituted into (5). Afterwards, the electric field $\boldsymbol{E}$ is expanded in terms of edge elements. One can obtain

$$
\{J\}_{\text {equ }}^{n+1}+\{J\}_{\text {equ }}^{n}=[\mathcal{R}]\left(\{e\}^{n+1}+\{e\}^{n}\right)
$$

where

$$
[\mathcal{R}]_{i j}=Y_{0} \int_{\partial \Omega}\left(\hat{n} \times \boldsymbol{W}_{i}^{(1)}\right) \cdot\left(\hat{n} \times \boldsymbol{W}_{j}^{(1)}\right) d \Omega .
$$

Finally, the first-order $\mathrm{ABC}$ can be easily implemented for the mixed FETD by substituting (11) into (6a).

\section{EQuivalences Between the MiXed FETD FORMULATIONS AND THE VW-FETD}

It is well-known that the second-order vector wave equation in a source-free region truncated with the first-order $A B C$ (7), expanded in terms of edge elements in space and discretized with the Newmark- $\beta$ method in time, can be written as

$$
\begin{aligned}
\{[\mathcal{M}] & \left.+\frac{\Delta t}{2}[\mathcal{R}]+\beta(\Delta t)^{2}[\mathcal{S}]\right\}\{e\}^{n+1} \\
= & \left\{2[\mathcal{M}]-(1-2 \beta)(\Delta t)^{2}[\mathcal{S}]\right\}\{e\}^{n} \\
& -\left\{[\mathcal{M}]-\frac{\Delta t}{2}[\mathcal{R}]+\beta(\Delta t)^{2}[\mathcal{S}]\right\}\{e\}^{n-1}
\end{aligned}
$$

where setting $\beta=0$ reduces it to the central difference method and $\beta=1 / 4$ results in an US time-marching [15]. $[\mathcal{S}]_{i j}=$ $\int_{\Omega} \mu^{-1}\left(\nabla \times \boldsymbol{W}_{i}^{(1)}\right) \cdot\left(\nabla \times \boldsymbol{W}_{j}^{(1)}\right) d \Omega$ is the stiffness matrix.

Having combined (6) and (11), the following equation can be obtained by eliminating the magnetic field variable:

$$
\begin{aligned}
\{[\mathcal{M}] & \left.+\frac{\Delta t}{2}[\mathcal{R}]\right\}\{e\}^{n+1} \\
= & \left\{2[\mathcal{M}]-(\Delta t)^{2}[\mathcal{C}]^{T}\left[\mathcal{M}_{f}\right][\mathcal{C}]\right\}\{e\}^{n} \\
& -\left\{[\mathcal{M}]-\frac{\Delta t}{2}[\mathcal{R}]\right\}\{e\}^{n-1} .
\end{aligned}
$$

In addition, it can be shown that $[\mathcal{S}]=[\mathcal{C}]^{T}\left[\mathcal{M}_{f}\right][\mathcal{C}]$ for Whitney elements. Hence, comparing (14) with (13) reveals that they are exactly the same for $\beta=0$. This equivalence, neglecting terms related to the $\mathrm{ABC}$ implementation, had been used to develop a stable hybrid FETD-FDTD [16].

If the average value of all undifferentiated quantities, instead of only $\left\{J_{e q u}\right\}$, is used in the discretization of (1), we reach

$$
\begin{aligned}
& {[\mathcal{M}] } \frac{\{e\}^{n+1}-\{e\}^{n}}{\Delta t} \\
&=[\mathcal{C}]^{T}\left[\mathcal{M}_{f}\right] \frac{\{b\}^{n+1}+\{b\}^{n}}{2}-\frac{\left\{J_{e q u}\right\}^{n+1}+\left\{J_{e q u}\right\}^{n}}{2} \\
& \frac{\{b\}^{n+1}-\{b\}^{n}}{\Delta t}=-[\mathcal{C}] \frac{\{e\}^{n+1}+\{e\}^{n}}{2}
\end{aligned}
$$

which is known as the Crank-Nicolson FETD (CN-FETD) [17]. Eliminating the magnetic field variable results in

$$
\begin{aligned}
\{[\mathcal{M}] & \left.+\frac{\Delta t}{2}[\mathcal{R}]+\frac{(\Delta t)^{2}}{4}[\mathcal{S}]\right\}\{e\}^{n+1} \\
= & \left\{2[\mathcal{M}]-\frac{(\Delta t)^{2}}{2}[\mathcal{S}]\right\}\{e\}^{n} \\
& -\left\{[\mathcal{M}]-\frac{\Delta t}{2}[\mathcal{R}]+\frac{(\Delta t)^{2}}{4}[\mathcal{S}]\right\}\{e\}^{n-1} .
\end{aligned}
$$

Clearly, this equation is exactly (13) with $\beta=1 / 4$. So, we can come to the conclusion that the LF-FETD and the CN-FETD are mixed versions of the VW-FETD discretized by the central-difference and the Newmark- $\beta$ with $\beta=1 / 4$, respectively. This may not only justify stability of the CN-FETD for arbitrary large time steps in numerical experiments [17], but also help to decrease dissipation of the US hybrid FETD-FDTD [18]. Furthermore, it shows that our approach to implement the $\mathrm{ABC}$ in the mixed FETD formulations is valid and leads to the same result as the VW-FETD truncated with that ABC. 


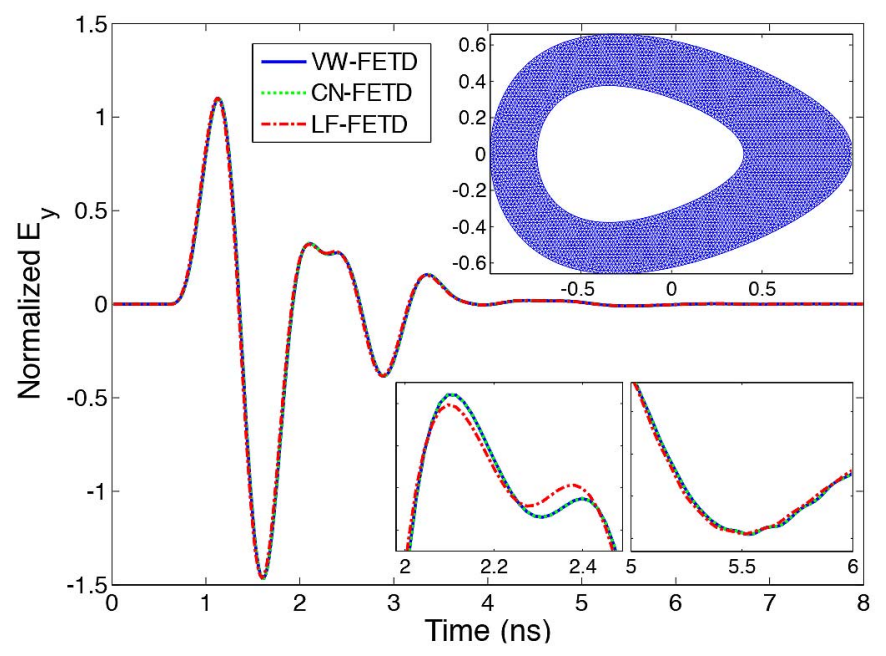

Fig. 1. Computational domain mesh, in meters, along with the normalized electric field recorded inside the domain obtained from the three different approaches.

It should be noted that the proposed approach is general and we believe that it can be utilized to implement high-order ABCs in the mixed FETD formulations. Particularly, in the case of a second-order Bayliss-Turkel ABC [19], simultaneous usage of both edge and face elements may lead to a more accurate implementation of the surface divergence term by adopting appropriate splitting of that term into normal and tangential components.

\section{RESULTS}

To validate the proposed approach, a simple 2D $\mathrm{TE}_{z}$ scattering problem has been considered. An egg-shaped PEC scatterer is illuminated with a magnetic line source placed at $(x, y)=(0.6 \mathrm{~m}, 0 \mathrm{~m})$ and excited with a differentiated Blackman-Harris pulse with the central frequency of $600 \mathrm{MHz}$. The domain has been truncated by another egg-shaped boundary. Triangular elements with the average edge length of $2.06 \mathrm{~cm}$ are utilized to discretize the computational domain, as shown in Fig. 1. The electric field along the y-axis $\left(E_{y}\right)$ has been recorded during the simulation at $(x, y)=(0.8 \mathrm{~m}, 0 \mathrm{~m})$. The time step is equal to $\Delta t=17.767 \mathrm{ps}$ which is $90 \%$ of the stability limit of the LF-FETD method. The problem has been solved using all three formulations, namely the VW-FETD discretized by the Newmark- $\beta$ method with $\beta=1 / 4$, the mixed CN-FETD and the mixed LF-FETD. The proposed approach is utilized to truncate the domain in the mixed formulations. The normalized $E_{y}$ 's obtained from three methods are depicted in Fig. 1. As can be seen, the CN-FETD and the VW-FETD have produced exactly identical results. Because it utilizes another time-stepping scheme, the LF-FETD solution is slightly different, but still quite close. These results also verify validity and performance of the proposed approach to implement the first-order $\mathrm{ABC}$ in the mixed FETD formulations.

\section{CONCLUSION}

A novel approach to implement the first-order $\mathrm{ABC}$ in the mixed FETD formulations has been proposed. The approach is based on placing a current source distribution on the boundary to cancel outgoing waves. Having shown the equivalence between the mixed FETD methods and the VW-FETD, the validity of the proposed approach has been upheld theoretically. Numerical results also confirm accuracy and validity of the formulations.

\section{REFERENCES}

[1] J. M. Jin and J. L. Volakis, "A hybrid finite element method for scattering and radiation by microstrip patch antennas and arrays residing in a cavity," IEEE Trans. Antennas Propagat., vol. 39, no. 11, pp. 1598-1604, Nov. 1991.

[2] A. Chatterjee, J. M. Jin, and J. L. Volakis, "Edge-based finite elements and vector ABC's applied to 3-D scattering," IEEE Trans. Antennas Propagat., vol. 41, no. 2, pp. 221-226, Feb. 1993.

[3] J.-M. Jin, The Finite Element Method in Electromagnetics, 2nd ed. New York: Wiley, 2002.

[4] D. Jiao, M. Lu, E. Michielssen, and J. M. Jin, "A fast time-domain finite element-boundary integral method for electromagnetic analysis," IEEE Trans. Antennas Propagat., vol. 49, pp. 1453-1461, Oct. 2001.

[5] D. Jiao, A. Ergin, B. Shanker, E. Michielssen, and J. M. Jin, "A fast time-domain higher-order finite element-boundary integral method for three-dimensional electromagnetic scattering analysis," IEEE Trans. Antennas Propagat., vol. 50, pp. 1192-1202, Sept. 2002.

[6] T. Rylander and J. M. Jin, "Perfectly matched layers for the time domain finite element method applied to Maxwell's equations," $J$. Comput. Physics, vol. 200, no. 1, pp. 238-250, Oct. 2004.

[7] S. Wang, R. Lee, and F. Teixeria, "Anisotropic-medium PML for vector FETD with modified basis functions," IEEE Trans. Antennas Propagat., vol. 54, pp. 20-27, Jan. 2006.

[8] B. Donderici and F. L. Teixeira, "Conformal perfectly matched layer for the mixed finite element time-domain method," IEEE Trans. Antennas Propagat., vol. 56, no. 4, pp. 1017-1026, Apr. 2008.

[9] A. Bayliss and E. Turkel, "Radiation boundary conditions for wave-like equations," Commun. Pure Appl. Math., vol. 33, pp. 707-725, 1980.

[10] A. Bayliss, M. Gunzburger, and E. Turkel, "Boundary conditions for the numerical solution of elliptic equations in exterior regions," SIAM J. Appl. Mat., vol. 42, no. 2, pp. 430-451, 1982.

[11] A. F. Peterson, "Absorbing boundary conditions for the vector wave equation," Microw. Opt. Technol. Lett., vol. 1, pp. 62-64, 1988.

[12] M. M. Botha and D. B. Davidson, "Rigorous, auxiliary variable-based implementation of a second-order ABC for the vector FEM," IEEE Trans. Antennas Propagat., vol. 54, pp. 3499-3504, Nov. 2006.

[13] F. L. Teixeira, "Time-domain finite-difference and finite-element methods for Maxwell equations in complex media," IEEE Trans. Antennas Propagat., vol. 56, no. 8, pp. 2150-2166, Aug. 2008.

[14] M. Wong, O. Picon, and V. F. Hanna, "A finite element method based on Whitney forms to solve Maxwell equations in the time domain," IEEE Trans. Magn., vol. 31, no. 3, pp. 1618-1621, May 1995.

[15] S. Gedney and U. Navsariwala, "An unconditionally stable finite element time-domain solution of the vector wave equation," IEEE Microwave Guided Wave Lett., vol. 5, pp. 332-334, Oct. 1995.

[16] T. Rylander and A. Bondeson, "Stable FEM-FDTD hybrid method for Maxwell's equations," Comput. Phys. Commun., vol. 125, pp. 75-82, Mar. 2000.

[17] M. Movahhedi, A. Abdipour, A. Nentchev, M. Dehghan, and S. Selberherr, "Alternating-direction implicit formulation of the finite-element time-domain method," IEEE Trans. Microw. Theory Tech., vol. 55, no. 6, pp. 1322-1331, June 2007.

[18] A. A. Sharbaf and R. Sarraf-Shirazi, "An unconditionally stable hybrid FETD-FDTD formulation based on the alternating-direction implicit algorithm," IEEE Antennas Wireless Propag. Lett., vol. 9, pp. 1174-1177, 2010.

[19] J. P. Webb and V. N. Kanellopoulos, "Absorbing boundary conditions for the finite element solution of the vector wave equation," Microw. Opt. Technol. Lett., vol. 2, pp. 370-372, 1988. 